\title{
Managing End-Stage Renal Disease in Older Patients: A Single Centre Experience with Renal Transplantation in the Elderly
}

\author{
Karsten Midtvedt, ${ }^{1}$ Kjersti Lønning, ${ }^{1}$ and Kristian Heldal ${ }^{2,3}$ \\ ${ }^{1}$ Section of Nephrology, Department of Organ Transplantation, Oslo University Hospital Rikshospitalet, 0424 Oslo, Norway \\ ${ }^{2}$ Clinic of Internal Medicine, Telemark Hospital, 3710 Skien, Norway \\ ${ }^{3}$ Faculty of Medicine, Institute of Clinical Medicine, University of Oslo, 0316 Oslo, Norway
}

Correspondence should be addressed to Kristian Heldal; hkri@sthf.no

Received 12 December 2013; Revised 24 February 2014; Accepted 24 February 2014; Published 25 March 2014

Academic Editor: Francois Madore

Copyright (C) 2014 Karsten Midtvedt et al. This is an open access article distributed under the Creative Commons Attribution License, which permits unrestricted use, distribution, and reproduction in any medium, provided the original work is properly cited.

\begin{abstract}
The increase of patients developing end-stage renal disease (ESRD) has occurred predominantly in the older adult population. As a consequence, the nephrologists will need to decide whom of these older patients are siutable for transplantation. There are very few absolute contraindications, such as active infection and recent malignancy, but there are many relative or potential contraindications in older patients. Worldwide, organs available for transplantation are limited. Some centers are reluctant to use organs from expanded criteria donors also in elderly recipients. This leads to long waiting lists and many older patients will die while they are waiting for an organ. It is vital that the patients who are accepted for renal transplantation are those who will derive most benefit, and correct selection of patients and donor organs is therefore of outmost importance. This paper describes the previous and planned research our research group has performed with focus on older renal transplant recipients with special emphasis on survival, basic immunosuppression, selection of organs, and health related quality of life.
\end{abstract}

\section{Introduction}

The increase of patients developing end-stage renal disease (ESRD) has occurred predominantly in the older adult population. During the last decade the annual incidence rate of ESRD in the USA for those 65 years and older has leveled off but continued to increase for those 75 years and older [1]. In Norway, a small country with just above 5 million inhabitants, a total of 515 new patients entered renal replacement therapy in 2012, that is, 102.6 per million inhabitants. Fifty percent were 65 years or older and a total of $68 \%$ were considered by their nephrologists to be a potential candidate for transplantation [2].

As the ESRD population gets older and older the nephrologists will need to decide whom of these older patients are siutable for transplantation. There are very few absolute contraindications, such as active infection and recent malignancy, but there are many relative or potential contraindications especially in older patients. In Norway, there has been no strict upper age limit and in principle transplantation is offered to all patients considered to profit from it. In 2012, 28\% of the recipients transplanted were above 65 years. In general, professional groups support renal transplantation for older patients with ESRD. The American Society of Transplantation guidelines on the evaluation of renal transplant candidates state that "there should be no absolute upper age limit for excluding patients whose overall health and life situation suggest that transplantation will be beneficial" [3]. The UK Renal Association guidelines state that "age is nor a contraindication to transplantation, but age related co-morbidity is an important limiting factor" [4]. The Canadian Society of Transplantation consensus guidelines state the same with "advanced age per se is not a contraindication to kidney transplantation" [5]. All guidelines suggest that physiologic age and burden of coexisting conditions are far more important than chronological age and that detailed evaluation and more aggressive screening in older recipients are warranted [3-5].

In patients with ESRD renal transplantation is accepted as the best alternative with the proviso that the patient is capable of tolerating surgery and the subsequent medical 
treatment $[6,7]$. It is associated with increased survival, is (when successful) less expensive, and provides a better health related quality of life (HRQOL) than lifelong dialysis $[6,8,9]$. When there is pronounced comorbidity, patients will in some cases only be offered conservative medical treatment aimed at delaying progression and relieving uraemia symptoms [10].

Worldwide, organs available for transplantation are however limited. This leads to long waiting lists which are increasing with $5-10 \%$ per year. The median wait for first renal transplantation within the Eurotransplant collaborative framework (Belgium, Luxembourg, the Netherlands, Slovenia, Germany, and Austria) was 50 months in 2012 [11] while the corresponding wait in Norway was 9 months [2]. The short transplant wait time is a consequence of relatively low incidence of ESRD in Norway and relatively good and stable availability of donor organs. At the end of 2013, there were 266 wait-listed patients in Norway. Twenty-two patients died on the waiting list and 13 were permanently removed. In Eurotransplant there were 10757 wait-listed patients. Five hundred and fifty-five died on the wait list and 803 were permanently removed. As shown, a significant number of patients will die while they are waiting for transplantation to be offered [12], or their general state of health may become so weakened that they are removed from the waiting list on medical grounds [13]. It is vital that the patients who are accepted for renal transplantation are those who will derive most benefit. There is therefore an ongoing debate on how to improve patient selection for transplantation. Age per se remains a relative contraindication. The surgical intervention is by itself associated with increased morbidity and mortality. In addition, there are side effects associated with life-long immunosuppression following renal transplantation. At our centre which is the only transplant centre in Norway, potential transplantation candidates are assessed according to a template which is age-independent. The assessment aims at excluding serious cardiovascular disease as well as cancer and other comorbidities, including mental illness and dementia. In addition, conditions that entail an unacceptably high risk in connection with the transplantation or posttransplantation must be eliminated. The assessment takes place locally and is headed by the patient's consultant nephrologist. Final approval of the individual case must be given by the transplantation community at Oslo University Hospital, Rikshospitalet.

As a consequence of the previously described short wait time, we have been able to perform a large number of renal transplantations to older recipients during the last 20 years. With the use of robust data from the Norwegian renal registry, we have been able to evaluate the results of these procedures.

Most data describing HRQOL after renal transplantation have been collected from average adult populations (median age $\sim 50$ years) [9]. Information on if/how HRQOL of older ESRD patients change during different phases of the treatment (dialysis, early posttransplantation and longterm transplantation) is virtually nonexistent. Neither are there, to our knowledge, any studies available describing the patients' expectations to the treatment nor the health economic aspects of transplantation of ESRD patients of advanced age. With today's improved dialysis treatment it is possible that many older patients with ESRD would profit from lifelong dialysis instead of renal transplantation. Life is, however, not just about quantity, that is, number of years, but also about quality. A vital question is, therefore, if renal transplantation in older patients adds life to years and not only years to life? Improved selection criteria are strongly warranted.

In older patients with ESRD there is often a coexisting high burden of comorbidities. The presence of cardiovascular disease (CVD) has been identified as a risk variable in adult renal transplant recipients [14]. In order to improve recipient selection, different comorbidity indexes have been developed. In a previous publication from our research group we found that in older recipients, a score at time of engraftment from the currently used Charlson Comorbidity Index did not predict posttransplant survival [15]. Recently a new and improved comorbidity index for patients with kidney diseases has been developed by Liu et al. [16] and from 2012 we have included this index as part of the routine workup of candidates for renal transplantation. In order to improve the selection criteria for transplant candidates, it will be of major interest to investigate if this index is associated with the survival and/or HRQOL outcomes after renal transplantation in a population of older patients.

In this paper, we will describe the previous research our research group has performed with focus on older renal transplant recipients. The following topics will be covered:

Section 2: Survival, Complications, and Risk Variables,

Section 3: Basic Immunosuppression,

Section 4: Selection of Organs,

Section 5: Health Related Quality of Life.

\section{Survival, Complications, and Risk Variables}

In their landmark report, Wolfe et al. showed that the average transplant recipients had a $68 \%$ decrease in mortality compared with those who remained on the wait list [7]. This survival advantage was, however, also seen in the older recipients. Patients, age 60-74 years, had a $61 \%$ lower mortality compared with age-matched patients on the wait list [7]. The majority of other previous studies investigating outcomes after renal transplantation in older recipients have been based on registry data from many different transplant centers reflecting several different transplantation protocols [17-19], or they have included a relatively low number of patients over the age of 70 [20]. Since we have been able to perform a relatively high number of transplantations in older patients this topic has been of major interest for us and we have addressed it in several single centre publications originating from our national transplant centre $[15,21,23,24]$. Initially we focused on patient and graft outcomes comparing results from 301 "elderly" renal transplant recipients $(\geq 70$ years of age) with 513 "senior" recipients (60-69 years of age) and a "control" group comprising 512 "average" adult renal recipients (age 45-54 years) [21]. A living donor transplantation was performed in $35 \%$ of patients: $17 \%$ in 
elderly recipients, $34 \%$ in senior recipients $(P<0.001)$, and $47 \%$ in control recipients $(P<0.001)$. Pre-emptive transplantation was performed in $19 \%$ of patients: $10 \%$ in elderly recipients, $18 \%$ in senior recipients $(P=0.003)$, and $25 \%$ in control recipients $(P<0.001)$. The elderly group had significantly lower rate of acute rejections during the first 12 weeks, compared with both the senior group $(P=0.005)$ and the control group $(P=0.002)$. Elderly and senior recipients had a higher incidence of death with a functioning graft during the follow-up: elderly $45 \%$, senior $31 \%(P<0.001)$, and control 13\% $(P<0.001)$. Five-year patient survival was $56 \%$ in the elderly group, $72 \%$ in the senior group $(P<0.001)$, and $91 \%$ in the control group $(P<0.001)$. Cardiovascular disease (31\% in the total material) and infection $(23 \%$ in the total material) were the most frequent causes of death in all age groups and the highest incidences were found in the elderly group (cardiovascular disease 34\%, infection $27 \%$ ). Five-year graft survival was 53\%, 70\%, and $84 \%$ in the elderly, senior, and control groups, respectively. There was, however, no difference in graft survival when censoring for death with functioning graft. Consequently, we drew the conclusion that the inferior graft survival in the elderly reflects a natural higher risk of death with functioning graft.

In the second paper [15] it was naturally to look closer at potentially relevant clinical parameters for patient survival, graft survival, and acute rejection in "elderly" ( $n=354)$, "senior" ( $n=577)$, and "control" $(n=563)$ recipients. Acute rejection during the first 90 days (HR 1.74 [1.342.25], $P<0.001$ ), time on dialysis before transplantation (HR 1.02 per month [1.01-1.03], $P<0.001$ ), and donor age $\geq 60$ years (HR 1.52 [1.14-2.01], $P=0.004$ ) were all associated with increased mortality in the elderly. Although comorbidity determined by the CCI score was not associated with increased mortality in the elderly group (HR 1.05 [0.981.12]), an association was found both in the senior (HR 1.17 per unit increase of the CCI score [1.08-1.27], $P<0.001$ ) and control groups (HR 1.33 [1.19-1.48], $P<0.001$ ). This was somewhat surprising knowing that $\mathrm{Wu}$ et al. described the CCI score as a significant predictor of survival in a cohort of 266 renal transplant recipients older than 60 years [20]. Their cohort was actually more similar to our senior group in which we also described an association. The explanation of the missing association may be that the threshold for being accepted as transplant candidate is higher with increasing age and that the CCI does not adequately deal with this possible selection bias. Alternatively, the comorbidity may be of less importance than age in this elderly cohort. Analyses of comorbidity in European patients requiring renal replacement therapy have indicated that even though comorbidity is an important predictor for mortality, the influence of comorbidity may be less important than expected when adjusted for confounders such as age, gender, primary renal disease, treatment modality, and country [22]. Delayed graft function (HR 3.69 [2.01-6.79], $P<0.001$ ), donor age $\geq 60$ years (HR 2.42 [1.30-4.49], $P=0.005$ ), and presence of human leukocyte antigen (HLA) antibodies (HR 3.96 [1.3811.37], $P=0.011$ ) were independently associated with deathcensored graft loss in the elderly. Treatment with azathioprine rather than mycophenolate moffetil (MMF), any HLA-A or
HLA-DR mismatch, donor age $\geq 60$ years, and presence of HLA antibodies were associated with increased risk for early acute rejections (the first 90 days after transplant) in all age groups.

The third paper compared the survival of older renal transplant recipients with similar aged patients who were accepted for transplantation but remained on dialysis [23]. All patients older than 70 years who started dialysis between 1990 and 2005 and were waitlisted for renal transplantation were included in the study $(N=286)$. The patients were categorised according to the year dialysis was started (19901999 versus 2000-2005). This categorisation was chosen because of the introduction in 2000 of a more potent immunosuppressive regimen which included replacement of azathioprine with MMF. Survival rates were analysed using a Kaplan-Meier model and a time-dependent Cox model. Comparisons were made between patients receiving a transplant and those who remained waitlisted. Patients starting dialysis between 1990 and 1999 had no significant long-term benefit of transplantation (HR for death 1.01 [0.581.75]). In contrast there was a substantial long-term benefit of transplantation among patients starting dialysis after 2000 (HR for death 0.40 [0.19-0.83], $P=0.014$ ). Although transplant recipients had an increased risk of death during the first year after transplantation (similar to Wolfe et al.), they had a long-term cumulative survival benefit compared to those remaining on dialysis. The median survival after transplantation increased from $3.7(3.0-4.4)$ years in the 1990-1999 cohort to $>6.7$ years in the 2000-2007 cohort. The early (first 90 days) rejection rate declined from $50 \%$ in the first era to $24 \%$ in the last era. For those who did not receive a renal transplant, the median survival after time of wait listing did not change between the two periods, 3.4 (3.3-3.7) years versus 3.1 (1.8-4.4) years. Similar methodology was used by Rao et al. in an elderly population with similar results as we have described [19]. Obviously, a randomized trial comparing transplantation versus dialysis will not be possible to perform because of ethical reasons. It must however be noticed that the time-dependent Cox model has several limitations compared to a prospective randomized control trial. The most important limitation is the selection of patients for transplantation. Since confounding variables were registered at the time of wait listing, it is impossible to control any confounders occurring after this time. However, as a result of a relatively short wait list in Norway, $80 \%$ of patients were eventually transplanted, indicating a relative low risk of selection bias.

As a consequence of this marked improvement of the long-term results after transplantation and reduction of incidence of early acute rejection episodes previously shown to be a strong risk variable, we wanted to reevaluate the specter of possible risk variables among patients receiving more potent immunosuppression. We therefore evaluated data in our transplant centre on all patients $(n=160)$ older than 70 years receiving their first renal transplant between 2000 and 2005 [24]. Survival data were analyzed using Cox proportional hazard models and the Kaplan-Meier method. The following factors were negatively associated with patients survival: age at transplantation; hazard ratio (HR) 1.09 per year [1.01-1.18], 
time on dialysis before transplantation; HR 1.29 per year [1.06-1.59] and presence of diabetes at transplantation; and HR 1.78 [1.00-3.16].Thirty-five patients (22\%) experienced an acute rejection episode during the first 90 days after transplant. Acute rejection episodes did not influence patient or graft survival. Patients with posttransplant pathological oral glucose tolerance test (OGTT) at 10 weeks after transplantation had significantly inferior survival compared to patients with normal OGTT. We concluded that in this low rejection risk population of older renal transplant recipients, age, time on dialysis before transplantation, concomitant diabetes mellitus, and development of a pathological OGTT 10 weeks after transplantation were associated with inferior patient survival [24].

\section{Basic Immunosuppression}

The human immune response is affected by the aging process. It is observed that older recipients develop fewer acute rejection episodes than younger recipients $[21,25,26]$, probably because of a less active immune system. Heavy immunosuppression in addition to a less active immune system may partly explain the dramatically increased number of deaths due to infections [26]. Some authors have therefore proposed that older recipients need less intense immunosuppressive treatment [27]. Less immunosuppression will lower the incidence of infections. On the other hand, acute rejection episodes have been shown to be a strong predictor of poor survival in older renal transplant recipients [15]. Because of age matching policies, older recipients will often receive organs from older donors. These organs may be more immunogenic due to nonspecific injuries that induce a proinflammatory milieu which, in turn, may activate immune responses [28-30] and high donor age has been found to increase the risk of acute rejection episodes in older recipients $[23,25]$. Organs from older donors are also likely to tolerate the trauma of transplantation (cold ischemia time, etc.) less well than organs from younger donors. In the Eurotransplant Senior Program, patients who received kidneys from older donors had significantly increased rate of acute rejections [30].

Currently, an interleukin 2 receptor antagonist (basiliximab) is recommended as first-line induction agent in low/normal risk recipients. No specific recommendations are made for older transplant recipients or recipients of older donor organs. We therefore performed a retrospective study based on prospectively collected registry data to investigate any association between induction treatment with basiliximab and improvement of graft survival and/or reduction of rejection episodes in recipients of kidneys from older deceased donors [31]. Data were retrieved for all recipients transplanted at our center from 2004 to 2009 with a kidney from a deceased donor aged $\geq 60$ years. The outcome was analyzed in multivariable Cox proportional hazard models adjusted for several confounding factors in addition to unadjusted Kaplan-Meier analyses. Results were compared between recipients treated with (IL-2 plus) or without (IL2 minus) basiliximab. All recipients received a calcineurin inhibitor, steroids, and MMF. A total of 232 first-transplant recipients were included (IL-2 plus $=149$, IL-2 minus $=$ 83). IL-2 minus was associated with increased risk of early acute rejection (OR 2.42; 95\% CI 1.25 to $4.68, P=0.009$ ) and steroid-resistant rejection (OR 8.04; 2.77 to $23.25, P<$ 0.001). IL-2 plus patients had superior two-year estimated uncensored ( $87 \%$ versus $70 \%, P=0.001)$ and death-censored (95\% versus 79\%, $P<0.001$ ) graft survival. We concluded that induction treatment with IL-2 receptor antagonist was associated with a reduction in acute rejection episodes and improvement of two-year graft survival in patients transplanted with kidneys from older deceased donors when compared to no induction. It must be noticed that the study had an observational design and accordingly no causality can be proven. Some centers advocate the use of ATG as induction also in elderly recipients. A direct comparison between induction with ATG and basiliximab was published in 2006 and showed that despite a lower incidence of acute rejection in the ATG group, there was no difference in the primary of the study, which was defined as a composite of acute rejection, delayed graft function, graft loss, and death [32]. Alemtuzumab [33] and rituximab [34] have been used for induction therapy in kidney transplantation and may be more frequently used in the future. In their paper from 2011, Gill et al. [35] examined the use of induction agents in recipients older than 60 years, transplanted in the USA between 2003 and 2008. A total of 7,140 patients received ATG, 6,215 received IL2RAB, and 1,465 received alemtuzumab.

They concluded that ATG may be preferable in highrisk recipients with high-risk donors and possibly in low-risk recipients with high-risk donors. In the remaining groups of recipients, the long-term outcomes did not appear to differ. Individual evaluation is important and our study establishes a hypothesis that should be further examined in randomized prospective trials.

No prospective trials examining maintenance immunosuppressive regimens in older renal transplant recipients have so far been published. We have previously published a study showing that the clearance of cyclosporine A (CsA) decreased with increasing age. In addition, we found that the older patients had a significant larger proportion of the whole blood CsA concentration located at the sight of action (within $\mathrm{T}$ lymphocytes). This indicates that in older recipients it might be safe to aim for an even lower "therapeutic target level" than current guidelines propose [36]. Currently we recommend tacrolimus/MMF as maintenance immunosuppressive agents in older recipients. This is in concordance with the recent findings from the US published by Gill et al. [35].

In the literature, treatment of rejections in older recipients is rarely addressed [37]. In treatment of biopsy-proven acute steroid-resistant cellular rejection, most centers use ATG [38]. The administration of ATG is associated with frequent infusion reactions and an increased incidence of infections and posttransplant lymphoproliferative disease [38]. Traditionally ATG has been administered in fixed dose for 3-14 days. We have previously published a study showing that individualized CD3-cell monitored ATG was safe and efficient leading to lower accumulative dose [39]. This approach may 
be of major importance in older recipients limiting the risk of being over immunosuppressed.

\section{Selection of Organs}

The overall population is aging. As an example, one may look at numbers from the US Census Bureau. They predict the number of Americans aged 65 and older to be 88.5 million in 2050. This is more than double the population of 40.2 million in 2010 [40]. In accordance with this, the number of people developing ESRD is anticipated to increase dramatically. As previously mentioned most groups support transplantation for older patients with ESRD and there is no scientific documentation that supports the introduction of an upper age limit for renal transplantation [17]. Organ shortage may, however, make it problematic to transplant older patients since they will "compete" for the same organs as younger recipients. When there is a lack of organs it is challenging to prioritize between older and younger patients on the waiting list. Often young patients, who potentially have many years to live ahead of them, will be selected. This trend has, however, become an extensive challenge for the transplant community as only a few lucky patients will receive a lifesaving organ, whereas the majority (especially older patients) will continue to wait for an appropriate organ until their death [1].

Use of living donors has been described as an important option, also for older transplant candidates [41], but even if this option was utilized maximally, the majority of patients would still have to wait for a deceased donor organ. One approach has been to expand the criteria for accepting kidneys from deceased donors. Different from a standard criteria deceased donor (SCD), an expanded criteria donor (ECD) is defined as a deceased donor older than 60 years or 50 to 59 years with at least two of three medical criteria: hypertension, cerebrovascular cause of death, or terminal serum creatinine immediately prior to organ recovery > $1.5 \mathrm{mg} / \mathrm{mL}$ [42]. This original ECD label included organs whose characteristics were associated with a relative risk of $>1.7$ for the outcome of overall graft loss (excluding paediatric donations) when compared to normotensive donors age 1039 years from the model generated by the Scientific Registry of Transplant Recipients (SRTR). Currently approximately 2000 ECD and 8000 SCD kidneys are transplanted annually in the USA [1]. Increased utilization of ECD would clearly increase the donor pool. In Norway, as a general rule, we consider organs from potential deceased donors with intact urine production and normal or only slightly elevated serum creatinine for kidney transplantation as single transplants for two recipients, even if the donor's estimated GFR at time of evaluation is below $60 \mathrm{~mL} / \mathrm{min}$. Thus, the key factors for allocation are anamnestic information of the donor's renal function prior to brain death and a maintained urine output in the ICU.

But are all ECD kidneys equal? The answer is obviously no. When addressing this topic, one must not, however, forget that the morbidity and mortality of the dialysis population are significant and the prognosis for older patients in dialysis is especially poor $[18,43]$. Older patients may spend the rest of their lives waiting for a deceased-donor kidney [44]. Some centres have made the argument to use donor age alone as a marker of quality. Schold et al. elegantly showed the wide range of quality in kidneys from deceased donors, where age was important but far from being the only "quality indicator" [45]. Some authors have claimed that a pretransplant histological quality evaluation of the potential transplant organ is mandatory. Depending on the score from this evaluation, one can decide whether the organ should be discarded or transplanted as a single or dual transplant [46, 47].

To evaluate our strategic approach of using very old donors for single transplants, we performed a retrospective quality control study [48]. The outcome of 54 single kidney transplantations from donors of more than 75 years (median 77.5 , range 75.2-86.1) was thoroughly assessed. Interestingly, the 1-, 3-, and 5-year corresponding death censored graft survival rates were $87 \%, 83 \%$, and $83 \%$ and the median serum creatinine at the end of follow-up was $163 \mu \mathrm{mol} / \mathrm{L}$ (range 103-348) after 23 months (range 6-144 months). In multivariable Cox regression analyses there we revealed a significant association between donor age and uncensored graft survival (HR 2.02 per year $(1.22-3.45) P=0.006$ ) but no association between donor age and uncensored graft survival (these results were not published in the original paper). The results indicate that the outcome of very old donor kidneys transplanted as single transplants are quite acceptable. An important factor for this favorable outcome is our protocol for optimal donor preservation and the logistics to minimize cold ischemia time. The finding is very important since, regrettably, many centers discard kidneys from elderly donors just because of age or with suspected marginal or inferior quality performing dual kidney transplantation to one recipient. Several centers even depend on histopathology examinations and histology scores of donor kidneys for their use, although biopsies have well-known inherent limitations because of sampling error and interobserver reproducibility. A consequence of such strategies is that the number of available kidneys for transplantation is reduced. Therefore, we have also evaluated the significance and the potential impact of histopathology evaluations by assessing all graft biopsies obtained at transplantation and throughout the first month after transplant in deceased donors over 75 years [48]. The biopsies were analyzed according to the Banff criteria, that is, glomerular sclerosis, tubular atrophy, interstitial fibrosis, and arteriosclerosis. Each parameter received a score ranging from 0 to 3 . The sum of the scores was defined as the global kidney score (GKS Banff), ranging from 0 to 15. As expected, there was a large individual variability in the magnitude of age-related histopathology parameters and a profound sampling variability between kidneys from the same donor. If histopathology score had been decisive for the use of these kidneys [47], a significant number of them would have been discarded or transplanted as dual grafts although they had excellent long-term function. With such criteria the number of available kidneys for transplantation may not be appropriately exploited. Given the knowledge that more than 30.000 patients are waiting for renal transplantation in Europe and approximately 100.000 patients in the USA [49], 


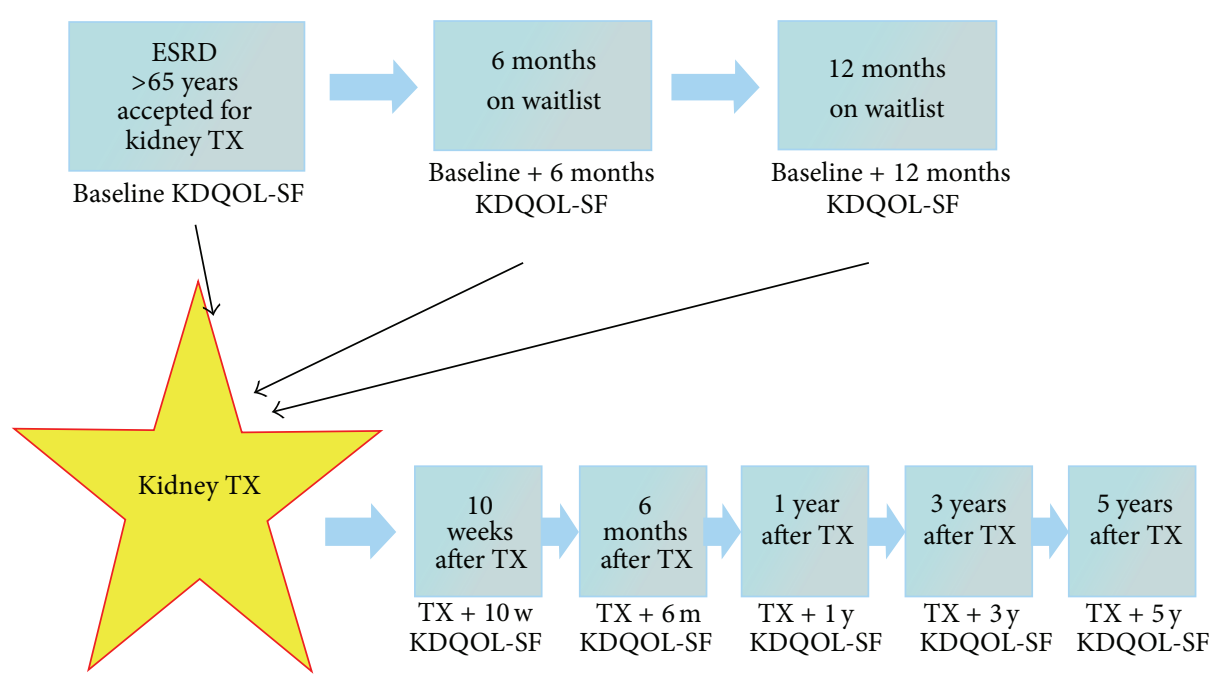

Figure 1: Study design Question 65.

it is our strong opinion that histopathology as decisive for the use of old donor kidneys in transplantation has major shortcomings and should be avoided. Our data could indicate that the use of old ECDs may be expanded more than today's practice. Over the last decade, age matching of donor kidneys has turned out to be of major importance for maximal utilization of deceased donor kidneys. It has been shown that proper age matching algorithms increase overall time with a functioning graft. It has also been shown that avoiding allocation of young donor kidneys to elderly recipients increases the overall function time of the grafts substantially [50]. In Norway, the recognition of the importance of age matching over other established allocation criteria has been fully implemented. Thus, when a very old donor is available, the age of the recipient is a primary criterion for selection and overrules HLA-matching. According to this policy, we have even transplanted kidneys from a 90-year-old deceased donor to recipients who were 71 and 79 years [51]. Both of them are still alive with good graft function almost five years after transplantation (personal message).

\section{Health Related Quality of Life}

Some of these older patients might live longer and better on continued dialysis. If we could detect these patients prior to transplantation, we could shorten the overall waiting time for transplantation and improve outcome for older patients with ESRD. To investigate this, our research group is currently performing a study entitled "Question 65." The aim of this study is to evaluate health related quality of life (HRQOL) among kidney transplant candidates older than 65 years of age at waitlisting/engraftment. The study is a longitudinal study following the patients from time accepted on the waiting list until 3-5 years after transplantation. HRQOL will be assessed by using KDQOL-SF-36 health survey [52]. Study design is described in Figure 1. To further evaluate the aspects of HRQOL in-depth interviews will be performed at baseline and one year after transplantation in 15-20 patients [53]. This

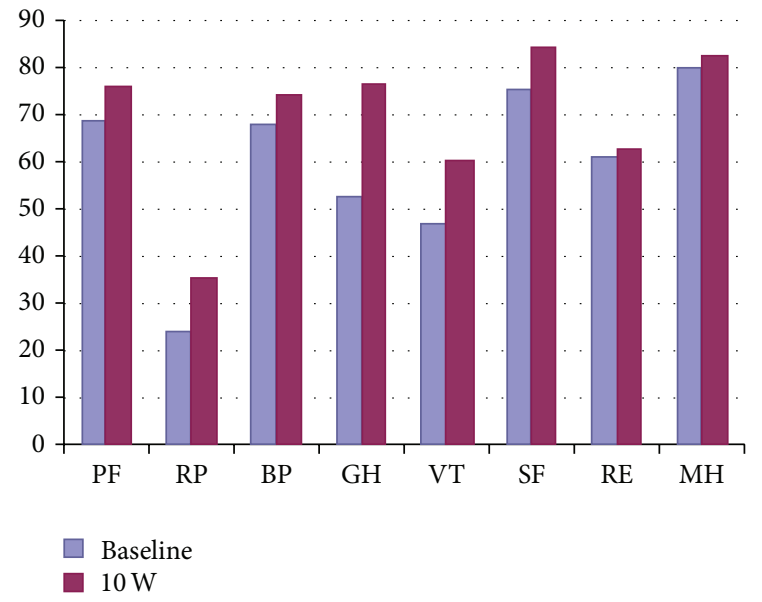

Figure 2: Preliminary SF-36 scores at baseline $(N=79)$ and 10 weeks after transplant $(N=26)$. PF: physical function, RP: role limitations caused by physical health problems, BP: bodily pain, $\mathrm{GH}$ : general health, VT: vitality, SF: social functioning, RE: role limitations caused by emotional problems, and ME: mental health. Scores per dimension range from 0 to 100 , and higher scores indicate better health related quality of life.

will also improve our understanding of the survey answers [54].

Inclusion started in January 2013. We aim to include 150200 patients and after one year of the planned two years inclusion period, a total of 106 patients are included and 43 of them are transplanted. We have performed a preliminary analysis of the data from the first 79 patients at baseline and 26 patients 10 weeks after transplant. Mean age at baseline was 71 years and 75\% were male. Preliminary SF-36 scores for baseline and 10 weeks after transplant are presented in Figure 2 and indicate an improvement after transplantation. The HRQOL score at 1 and 3 years after transplant will however be even more important. Are these improvements permanent or will 
negative side effects of immunosuppressive drugs dominate more and more? Hopefully, this study will provide novel information about the effect of renal transplantation on HRQOL in older recipients and thereby help clinicians in the choice between continued dialysis and transplantation for elderly patients with ESRD.

\section{Concluding Remarks}

Most publications regarding transplantation in older patients are favourable. One must, however, not forget that patients that make it to transplantation are a highly selected group of individuals. The selection bias must be considered when deciding on transplantation in older patients. Publications covering older recipients focus on transplant survival, patient survival, infections, and rejections. Older patients have a limited reserve and complications following transplantation can lead to loss of mobility, cognitive decline, and even loss of independence. These are posttransplantation outcomes that no one wants. Further research on criteria for the optimal use of ECDs is important because both the recipient and the potential donor pool are becoming older and the numbers of patients on the waiting list are increasing. Our group will continue to perform research and evaluations to help improve selection of older patients with ESRD whom will benefit most from renal transplantation.

\section{Conflict of Interests}

The authors declare that there is no conflict of interests regarding the publication of this paper.

\section{Acknowledgment}

The authors have not received any external financial support for the preparation of this paper.

\section{References}

[1] A. J. Collins, R. N. Foley, C. Herzog et al., "US renal data system 2012 annual data report," The American Journal of Kidney Diseases, vol. 61, no. 1, supplement 1, p. A7, 2013.

[2] T. Leivestad, "Annual report Norwegian renal registry 2012," 2013, http://www.nephro.no/nnr.html.

[3] B. L. Kasiske, C. B. Cangro, S. Hariharan et al., "The evaluation of renal transplant candidates: clinical practice guidelines," The American Journal of Transplantation, vol. 1, no. 2, pp. 5-95, 2001.

[4] C. Dudley and P. Harden, Assessment of the Potential Kidney Transplant Recipient, The Renal Association, 2011, http:// www.renal.org/Clinical/GuidelinesSection/AssessmentforRenalTransplantation.aspx.

[5] G. Knoll, S. Cockfield, T. Blydt-Hansen et al., "Canadian society of transplantation consensus guidelines on eligibility for kidney transplantation," Canadian Medical Association Journal, vol. 173, no. 10, pp. S1-S25, 2005.

[6] N. Kontodimopoulos and D. Niakas, "An estimate of lifelong costs and QALYs in renal replacement therapy based on patients' life expectancy," Health Policy, vol. 86, no. 1, pp. 85-96, 2008.
[7] R. A. Wolfe, V. B. Ashby, E. L. Milford et al., "Comparison of mortality in all patients on dialysis, patients on dialysis awaiting transplantation, and recipients of a first cadaveric transplant," The New England Journal of Medicine, vol. 341, no. 23, pp. 17251730, 1999.

[8] D. Niakas and N. Kontodimopoulos, "Is renal transplantation the most cost-effective and preferable therapy for patients suffering from end-stage renal disease or not?" Health Policy, vol. 89, no. 3, pp. 329-331, 2009.

[9] P. Rebollo, F. Ortega, J. M. Baltar et al., "Health related quality of life (HRQOL) of kidney transplanted patients: variables that influence it," Clinical Transplantation, vol. 14, no. 3, pp. 199-207, 2000.

[10] F. E. M. Murtagh, J. E. Marsh, P. Donohoe, N. J. Ekbal, N. S. Sheerin, and F. E. Harris, "Dialysis or not? A comparative survival study of patients over 75 years with chronic kidney disease stage 5," Nephrology Dialysis Transplantation, vol. 22, no. 7, pp. 1955-1962, 2007.

[11] “Euorotransplant annual report 2012," 2013, http://www.eurotransplant.nl/?id=annual_report.

[12] K. K. Stevens, Y. M. Woo, M. Clancy, J. D. McClure, J. G. Fox, and C. C. Geddes, "Deceased donor transplantation in the elderly-are we creating false hope?” Nephrology Dialysis Transplantation, vol. 26, no. 7, pp. 2382-2386, 2011.

[13] J. D. Schold, T. R. Srinivas, A. R. Sehgal, and H. U. MeierKriesche, "Half of kidney transplant candidates who are older than 60 years now placed on the waiting list will die before receiving a deceased-donor transplant," Clinical Journal of the American Society of Nephrology, vol. 4, no. 7, pp. 1239-1245, 2009.

[14] E. Petersen, B. C. Baird, F. Shihab et al., "The impact of recipient history of cardiovascular disease on kidney transplant outcome," ASAIO Journal, vol. 53, no. 5, pp. 601-608, 2007.

[15] K. Heldal, A. Hartmann, T. Leivestad et al., "Clinical outcomes in elderly kidney transplant recipients are related to acute rejection episodes rather than pretransplant comorbidity," Transplantation, vol. 87, no. 7, pp. 1045-1051, 2009.

[16] J. Liu, Z. Huang, D. T. Gilbertson, R. N. Foley, and A. J. Collins, "An improved comorbidity index for outcome analyses among dialysis patients," Kidney International, vol. 77, no. 2, pp. 141-151, 2010.

[17] E. Huang, N. Poommipanit, M. S. Sampaio et al., "Intermediateterm outcomes associated with kidney transplantation in recipients 80 years and older: an analysis of the OPTN/UNOS database," Transplantation, vol. 90, no. 9, pp. 974-979, 2010.

[18] S. K. Munshi, N. Vijayakumar, N. A. Taub, H. Bhullar, T. C. N. Lo, and G. Warwick, "Outcome of renal replacement therapy in the very elderly," Nephrology Dialysis Transplantation, vol. 16, no. 1, pp. 128-133, 2001.

[19] P. S. Rao, R. M. Merion, V. B. Ashby, F. K. Port, R. A. Wolfe, and L. K. Kayler, "Renal transplantation in elderly patients older than 70 years of age: results from the scientific registry of transplant recipients," Transplantation, vol. 83, no. 8, pp. 10691074, 2007.

[20] C. Wu, R. Shapiro, H. Tan et al., "Kidney transplantation in elderly people: the influence of recipient comorbidity and living kidney donors," Journal of the American Geriatrics Society, vol. 56, no. 2, pp. 231-238, 2008.

[21] K. Heldal, T. Leivestad, A. Hartmann, M. V. Svendsen, B. H. Lien, and K. Midtvedt, "Kidney transplantation in the elderlythe Norwegian experience," Nephrology Dialysis Transplantation, vol. 23, no. 3, pp. 1026-1031, 2008. 
[22] J. G. van Manen, P. C. W. van Dijk, V. S. Stel et al., "Confounding effect of comorbidity in survival studies in patients on renal replacement therapy," Nephrology Dialysis Transplantation, vol. 22, no. 1, pp. 187-195, 2007.

[23] K. Heldal, A. Hartmann, D. C. Grootendorst et al., "Benefit of kidney transplantation beyond 70 years of age," Nephrology Dialysis Transplantation, vol. 25, no. 5, pp. 1680-1687, 2010.

[24] K. Heldal, A. Hartmann, T. Leivestad, A. Foss, and K. Midtvedt, "Risk variables associated with the outcome of kidney recipients $>70$ years of age in the new millennium," Nephrology Dialysis Transplantation, vol. 26, no. 8, pp. 2706-2711, 2011.

[25] D. McKay and J. Jameson, "Kidney transplantation and the ageing immune system," Nature Reviews Nephrology, vol. 8, no. 12, pp. 700-708, 2012.

[26] H. U. Meier-Kriesche, A. Ojo, J. Hanson et al., "Increased immunosuppressive vulnerability in elderly renal transplant recipients," Transplantation, vol. 69, no. 5, pp. 885-889, 2000.

[27] M. Badowski, C. Gurk-Turner, C. Cangro et al., "The impact of reduced immunosuppression on graft outcomes in elderly renal transplant recipients," Clinical Transplantation, vol. 23, no. 6, pp. 930-937, 2009.

[28] J. W. de Fijter, M. J. K. Mallat, I. I. N. Doxiadis et al., "Increased immunogenicity and cause of graft loss of old donor kidneys," Journal of the American Society of Nephrology, vol. 12, no. 7, pp. 1538-1546, 2001.

[29] R. Oberhuber, X. Ge, and S. G. Tullius, "Donor age-specific injury and immune responses," The American Journal of Transplantation, vol. 12, no. 1, pp. 38-42, 2012.

[30] J. Pratschke, V. Merk, A. Reutzel-Selke et al., "Potent early immune response after kidney transplantation in patients of the European senior transplant program," Transplantation, vol. 87, no. 7, pp. 992-1000, 2009.

[31] K. Heldal, S. Thorarinsdottir, A. Hartmann et al., "Induction with interleukin-2 antagonist for transplantation of kidneys from older deceased donors: an observational study, Transplantation Research, vol. 2, article 11, 2013.

[32] D. C. Brennan, J. A. Daller, K. D. Lake, D. Cibrik, and D. Del Castillo, "Rabbit antithymocyte globulin versus basiliximab in renal transplantation," The New England Journal of Medicine, vol. 355, no. 19, pp. 1967-1977, 2006.

[33] R. D. Morgan, J. M. O'Callaghan, S. R. Knight, and P. J. Morris, "Alemtuzumab induction therapy in kidney transplantation: a systematic review and meta-analysis," Transplantation, vol. 93, no. 12, pp. 1179-1188, 2012.

[34] G. Tyden, H. Ekberg, G. Tufveson, and L. Mjornstedt, "A randomized, double-blind, placebo-controlled study of single dose rituximab as induction in renal transplantation: a 3-year follow-up," Transplantation, vol. 94, no. 3, pp. e21-e22, 2012.

[35] J. Gill, M. Sampaio, J. S. Gill et al., "Induction immunosuppressive therapy in the elderly kidney transplant recipient in the United States," Clinical Journal of the American Society of Nephrology, vol. 6, no. 5, pp. 1168-1178, 2011.

[36] P. Falck, A. Åsberg, K. Byberg et al., "Reduced elimination of cyclosporine a in elderly ( $>65$ years) kidney transplant recipients," Transplantation, vol. 86, no. 10, pp. 1379-1383, 2008.

[37] K. Heldal and K. Midtvedt, "Managing transplant rejection in the elderly: the benefits of less aggressive immunosuppressive regimens," Drugs and Aging, vol. 30, no. 7, pp. 459-466, 2013.

[38] M. W. van den Hoogen, A. J. Hoitsma, and L. B. Hilbrands, "Anti-T-cell antibodies for the treatment of acute rejection after renal transplantation," Expert Opinion on Biological Therapy, vol. 12, no. 8, pp. 1031-1042, 2012.
[39] K. Midtvedt, P. Fauchald, B. Lien et al., "Individualized T cell monitored administration of ATG versus OKT3 in steroidresistant kidney graft rejection," Clinical Transplantation, vol. 17, no. 1, pp. 69-74, 2003.

[40] G. Vincent and V. Velkoff, "The next four decades. The older population in the United States: 2010 to 2050 population estimates and projections," US Department of Commerce Economics and Statistics Administration, 2010, http:// www.census.gov/prod/2010pubs/p25-1138.pdf.

[41] M. Cooper and C. L. Forland, "The elderly as recipients of living donor kidneys, how old is too old?" Current Opinion in Organ Transplantation, vol. 16, no. 2, pp. 250-255, 2011.

[42] F. K. Port, J. L. Bragg-Gresham, R. A. Metzger et al., "Donor characteristics associated with reduced graft survival: an approach to expanding the pool of kidney donors," Transplantation, vol. 74, no. 9, pp. 1281-1286, 2002.

[43] C. M. Mandigers, W. de Jong, A. W. L. van den Wall Bake, and P. G. Gerlag, "Renal replacement therapy in the elderly," Netherlands Journal of Medicine, vol. 49, no. 4, pp. 135-142, 1996.

[44] K. Heldal and K. Midtvedt, "Doctor, should I remain on dialysis or accept the ECD kidney offered to me?" The American Journal of Kidney Diseases, vol. 59, no. 6, pp. 748-750, 2012.

[45] J. D. Schold, B. Kaplan, R. S. Baliga, and H. U. Meier-Kriesche, "The broad spectrum of quality in deceased donor kidneys," The American Journal of Transplantation, vol. 5, no. 4, part 1, pp. 757$765,2005$.

[46] G. Remuzzi, J. Grinyò, P. Ruggenenti et al., "Early experience with dual kidney transplantation in adults using expanded donor criteria," Journal of the American Society of Nephrology, vol. 10, no. 12, pp. 2591-2598, 1999.

[47] G. Remuzzi, P. Cravedi, A. Perna et al., "Long-term outcome of renal transplantation from older donors," The New England Journal of Medicine, vol. 354, no. 4, pp. 343-352, 2006.

[48] A. Foss, K. Heldal, H. Scott et al., "Kidneys from deceased donors more than 75 years perform acceptably after transplantation," Transplantation, vol. 87, no. 10, pp. 1437-1441, 2009.

[49] United Network for Organ Sharing (UNOS), UNOS, 2013, http://www.unos.org/.

[50] W. H. Lim, S. Chang, S. Chadban et al., "Donor-recipient age matching improves years of graft function in deceased-donor kidney transplantation," Nephrology Dialysis Transplantation, vol. 25, no. 9, pp. 3082-3089, 2010.

[51] K. Midtvedt, T. Namtvedt, H. Scott et al., "Single transplanted kidneys from a 90-year-old deceased donor perform acceptably at 1 year," Transplantation Proceedings, vol. 43, no. 5, pp. 21072109, 2011.

[52] R. D. Hays, J. D. Kallich, D. L. Mapes, S. J. Coons, and W. B. Carter, "Development of the kidney disease quality of life (KDQOL(TM)) instrument," Quality of Life Research, vol. 3, no. 5, pp. 329-338, 1994.

[53] A. Tong, J. R. Chapman, A. Israni, E. J. Gordon, and J. C. Craig, "Qualitative research in organ transplantation: recent contributions to clinical care and policy," The American Journal of Transplantation, vol. 13, no. 6, pp. 1390-1399, 2013.

[54] K. Malterud, "Qualitative research: standards, challenges, and guidelines," The Lancet, vol. 358, no. 9280, pp. 483-488, 2001. 


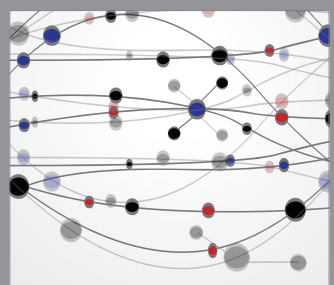

The Scientific World Journal
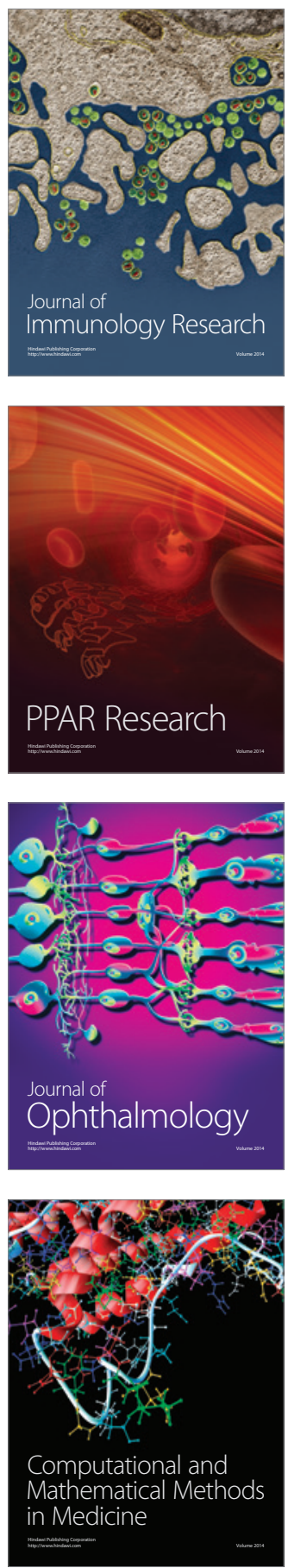

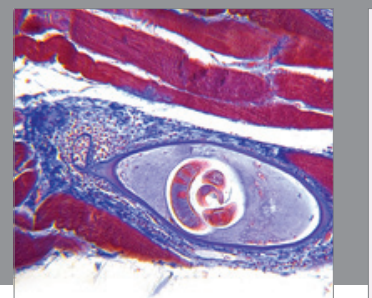

Gastroenterology

Research and Practice
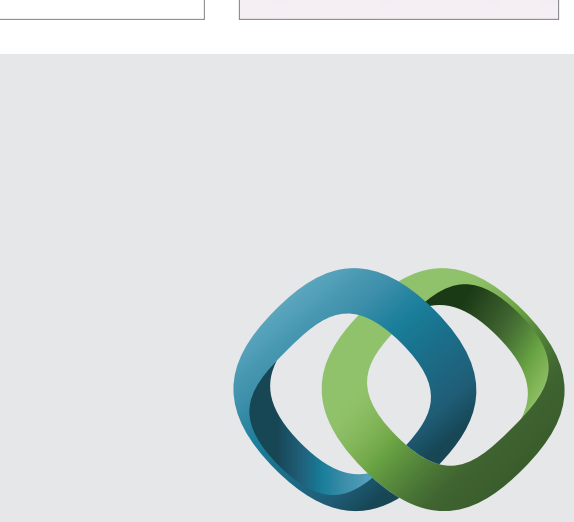

\section{Hindawi}

Submit your manuscripts at

http://www.hindawi.com
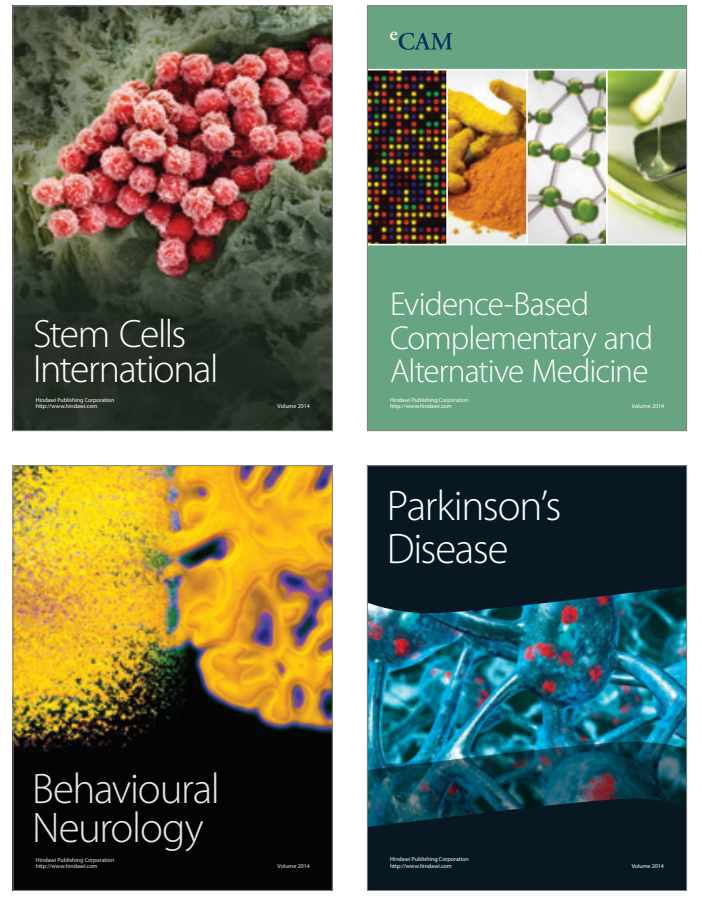
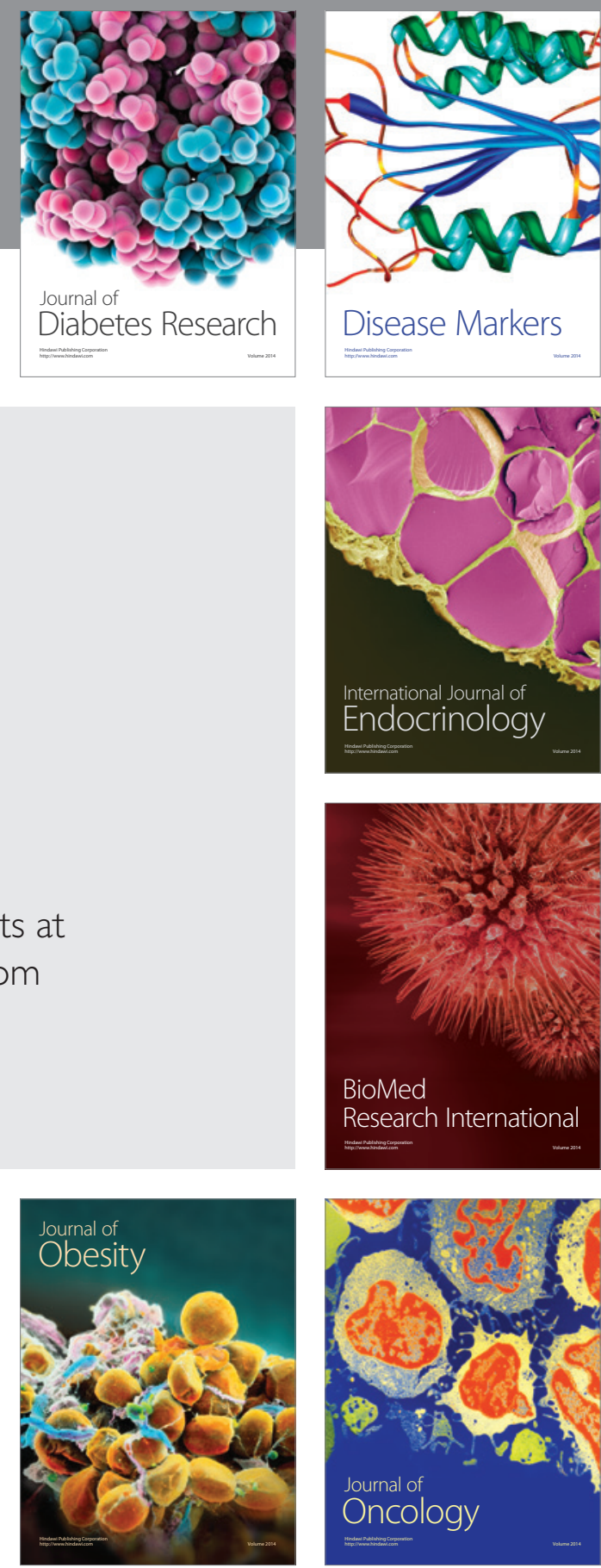

Disease Markers
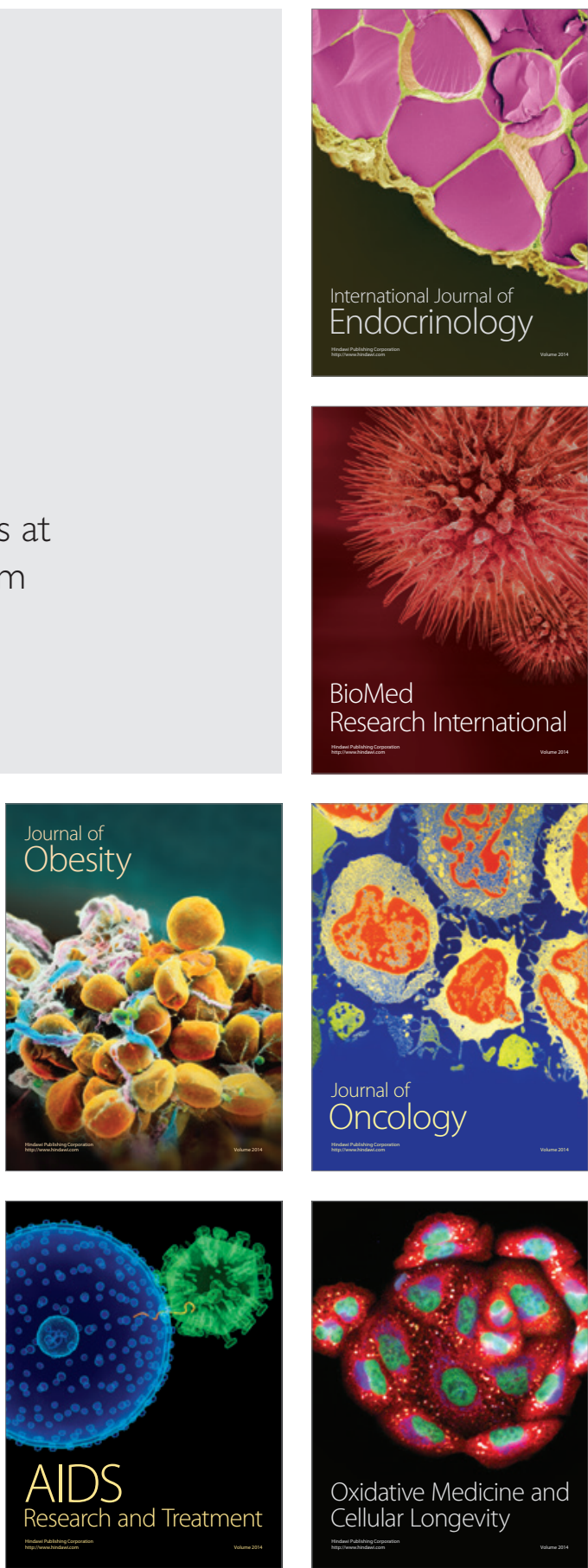